\title{
A numerical study of the scalar field in turbulent round jet with co-flowing stream
}

\section{H. Gazzah, H. Belmabrouk, M. Sassi}

The last sentence of the Abstract should read: "It is mainly shown that the co-flow reduces the jet spreading rate, but on the other hand decreases the mixing efficiency."

The last sentence of Sect. 4 should read: "It is obvious that the co-flow decreases considerably the mixing efficiency."

The labels in Fig. 8 were incorrect in the published article. The corrected figure is printed here:

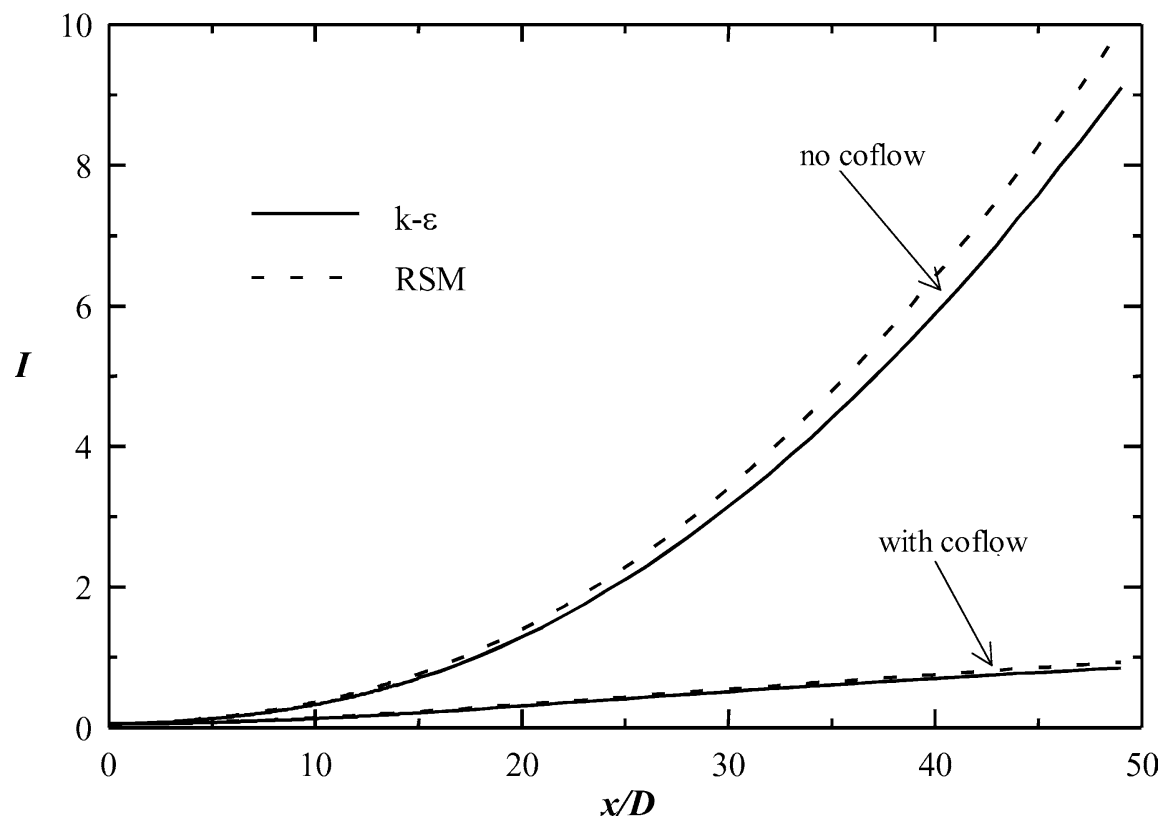

Fig. 8. Evolution of the axial mixing efficiency

The online version of the original article can be found at http://dx.doi.org/10.1007/s00466-004-0587-8

Published online: 10 September 2004

M. H. Gazzah ( $₫)$, H. Belmabrouk

Département de Physique, Faculté des Sciences de Monastir, avenue de I'environnement 5019 Monastir, Tunisie

e-mail: Hichem.Gazzah@fsm.rnu.tn

M. Sassi

Départment de Mécanique, Ecole Nationale d'Ingénieurs de Tunis, B.P. 37, 1002 Belvédère Tunis, Tunisie 